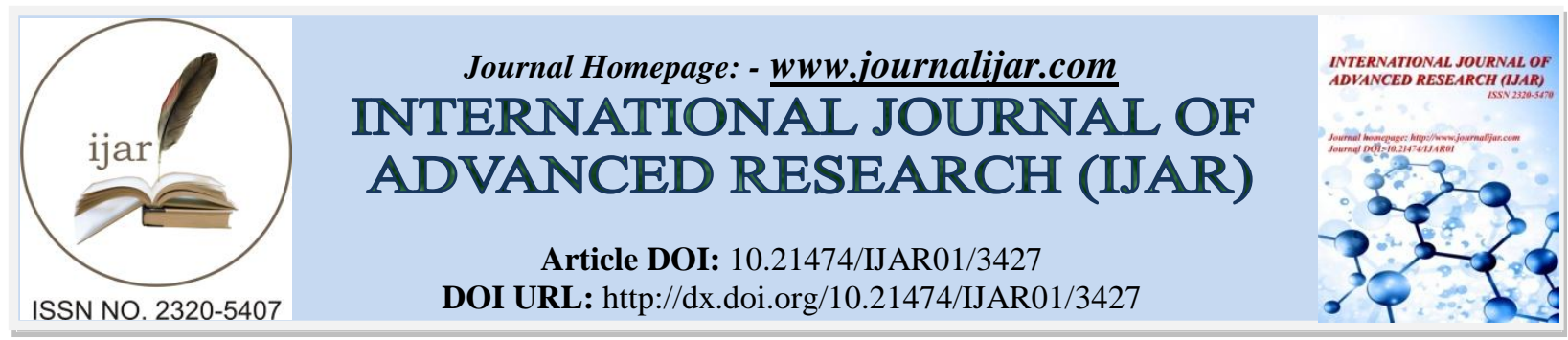

RESEARCH ARTICLE

\title{
ENZYME ACTIVITY PROFILING OF EXO - $\beta$-1,4- GLUCANASE, ENDO- $\beta$-1,3- GLUCANASE AND PROTEASE IN STREPTOMYCES SPECIES FROM HIGHLAND, MIDLAND AND LOWLAND AREAS OF KERALA, INDIA.
}

\section{Lekshmi K. Edison ${ }^{1}$, Reshma R. Anilkumar ${ }^{1}$, Gayathri V. ${ }^{2}$ and Pradeep N. S. ${ }^{1 *}$}

1. Division of Microbiology, Jawaharlal Nehru Tropical Botanical Garden and Research Institute, Palode, Thiruvananthapuram, 695562 Kerala, India.

2. Department of Biotechnology and Biochemical Engineering, SCT College of Engineering, Thiruvananthapuram, 695518 Kerala, India.

\section{Manuscript Info}

Manuscript History

Received: 12 December 2016

Final Accepted: 16 January 2017

Published: February 2017

Enzyme activity, Land use type, Number of isolates, Soil quality
Key words:-

\begin{abstract}
Variability in land use have substantial influence on biological properties of soil that persistently associates with soil quality, evaluated by microbial and biochemical indicators in soil. Kerala, located in the southwestern tip of India, geographically divided into eastern highland, central midland and western lowland. The natural classification of Kerala's landmass is an excellent illustration for describing land use types and environmental changes. This study scrutinizes the comparative activities of exo- $\beta$-1,4-glucanase, endo- $\beta$-1,3-glucanase and protease in Streptomyces spp. from different highland, midland and lowland areas of Kerala. Soils collected from 7 different locations of each land area were analyzed for estimating enzyme activity by semiquantitative and quantitative approaches. A significant fluctuation in exo- $\beta-1,4$-glucanase activity was observed in three land use systems, showed high activity in highland areas where natural vegetation occurs and significantly decreased to lowland regions, mostly arable areas. Number of Streptomyces isolates also revealed a decreasing trend from highland to lowland areas. $\mathrm{pH}$, electrical conductivity (EC), soil organic carbon (SOC) and soil organic matter (SOM) were showed a significant correlation with the number of Streptomyces isolates. The variation of exo- $\beta-1,4$-glucanase activity from highland to lowland suggested the sensitivity of the enzyme as an ecological and a soil quality indicator, hence it can be used as a conspicuous indicator of environmental changes.
\end{abstract}

Copy Right, IJAR, 2017,. All rights reserved.

\section{Introduction:-}

Soil microbial biomass and their enzyme activities in soil are very sensitive to environmental stresses and changes in soil management practices. The exposure of soil to degradation primarily affected its biological properties and diminishes the productive capacities. Enzyme activities in soils are the useful contenders, which provides information about soil ecological stress linking soil degradation (Yang et al., 2012). Rigorous anthropogenic activities, primarily land changes, from forest to intensive agriculture, causing severe consequence on soil quality and drastic health problems in plants (Matson et al., 1997). Microbial enzymes that mediate the biogeochemical

Corresponding Author:- Pradeep N. S.

Address:- Division of Microbiology, Jawaharlal Nehru Tropical Botanical Garden and Research Institute, 2431 
cycles, in addition, promptly responds to environmental signals considered as a sensitive indicators of environmental variations and soil qualities (Schloter et al., 2003). In addition, it can also be used as active measures of soil productivity and effect of pollutants (Tate, 1995). In recent years, there have been a growing interest in the exploration of microbial diversity as a tool for indicating environmental and ecosystem changes.

Conversion of natural ecosystem to arable land masses by human interventions intensify the use chemical fertilizers and pesticides (Matson et al., 1997). It extremely affect soil biota, by changing their population and biochemical activities. Moreover, soil mineralization is accomplished by the metabolic and enzyme activities of soil microorganisms. The soil enzyme activities are sensitive to soil biochemical characters like $\mathrm{pH}$, temperature, electrical conductivity, soil water potential etc. Considering the environmental significance of exo - $\beta$-1,4-glucanase, endo- $\beta$-1,3-glucanase and protease, these three were incorporated in the scenario. Exo- $\beta$ - 1,4 -glucanase and endo- $\beta$ 1,3-glucanase are cellulosome complex enzymes (Zhang and Zhang 2013). The activities of $\beta$-glucanases and protease in soil are regulated by many factors like climate, organic matters and soil properties. The modulation of these enzyme activities in different ecosystems by the input of pollutants and the impact of climate change, requires investigation (Sinegani and Mahohi, 2010; Vranova et al., 2013).

Streptomyces communities are the most fascinating members in the world of microbial diversity. They are most cherished organisms with great research interest due to high pharmacological and commercial concern. They are filamentous Gram positive soil bacteria, contribute significantly to the turnover of complex biopolymers such as lignocellulose, hemicellulose, pectin, keratin, and chitin. Subsequently there is vital evidence accessible due to the introduction of genome and protein sequencing data, Streptomyces spp. has been continuously screened for the production of various enzymes (Sharma, 2014). They are also serving as good models for the production of vital metabolites and enzymes with respect to stress response. Many evidences indicated that Streptomyces spp. have been isolated from diverse habitats like deserts (Hozzein et al., 2008), marine (Valli et al., 2012), protected forest areas (Thakur et al., 2007) etc. Very less information is available regarding variation of Streptomyces isolates between different geological land areas.

Kerala is located at the south- western corner of Peninsular India, between $8^{0} 17^{\prime} 30^{\prime \prime} \mathrm{N}$ and $12^{0} 47^{\prime} 40^{\prime \prime} \mathrm{N}$ north latitudes and $74^{0} 27^{\prime} 47^{\prime \prime} \mathrm{E}$ and $77^{\circ} 37^{\prime} 12^{\prime \prime} \mathrm{E}$ east longitudes. The geographical landscape of Kerala exhibits high variability in distribution of the landmass. Based on the physical features of Kerala, its total landmass naturally categorized into three geographical zones namely high land, mid land and low land. These regions run parallel to each other from northern end to southern end of the state. Highlands are the mountain ranges located in the eastern ends mainly sloping down from the Western Ghats. Lowlands are the unique agricultural areas in Kerala that cover coastal areas, back waters, lagoons and river deltas. Major parts of low land areas lies below the sea level. Midland is located between highland and lowland, characterized by undulating hills and valleys. This study scrutinizes the enzyme activity profiling of exo- $\beta-1,4$-glucanase, endo- $\beta-1,3$-glucanase and protease in Streptomyces spp. in different highland, midland and lowland areas.

\section{Materials and Methods:- \\ Site description and soil sampling:-}

The investigated sample sites were widely distributed in three geographical zones of Kerala, namely highland, midland and lowland areas (Figure 1). Seven different locations were selected from each land areas. Highland soil samplings were done in Western Ghats areas like Chinnar, Munnar, Marayur, Anaimalai, Nelliyampathy, Agasthyakoodam and Wayanad. Midland areas were Bonacaud, Palode, Kallar, Neryamangalam, Kothamangalam, Ottapalam and Thaliparamba. Lowland areas represented primarily paddy fields from different locations namely Kainakkari, Puthupalli, Mankombu, Changanasseri, Nedungad, Mavelikkara and Thiruvalla. The location, elevation, land use type etc were provided in Table 1. In each location, three sampling points were randomly apportioned at a distance of $30 \mathrm{~m}$ and upper organic layer of soil approximately 5 to 10 centimeters of depth were aseptically taken by an auger.

\section{Enrichment, isolation and purification of Streptomyces:-}

Soil samples were mixed with $1 \% \mathrm{CaCl}_{2}$ and incubated at $28^{\circ} \mathrm{C}$ for 10 days before use for enrichment. Standard dilution plate technique was followed for the isolation of Actinomycetes. For each sample, one gram soil was oven dried and suspended in $9 \mathrm{~mL}$ sterile distilled water and thoroughly vortexed for $30 \mathrm{~min}$ at $150 \mathrm{rpm}$ at room temperature. The suspension was serially diluted to obtain $10^{-1}$ to $10^{-6}$ dilutions. $1.0 \mathrm{~mL}$ of $10^{-3}$ to $10^{-6}$ dilutions were plated on Inorganic salt-starch agar (ISP-4) media (Starch,10g; $\mathrm{K}_{2} \mathrm{HPO}_{4}, 1 \mathrm{~g} ; \quad \mathrm{MgSO}_{4} .7 \mathrm{H}_{2} \mathrm{O}, 1 \mathrm{~g} ; \mathrm{NaCl}, 1 \mathrm{~g}$; 
$\left(\mathrm{NH}_{4}\right)_{2} \mathrm{SO}_{4}, 2 \mathrm{~g} ; \mathrm{CaCO}_{3}, 2 \mathrm{~g}$ and Trace salt solution, $\left.1 \mathrm{ml}\right)$. After incubation of 7 days at $28^{0} \mathrm{C}$ Streptomyces like isolates were selected randomly based on their morphology. Number of total Streptomyces colonies were evaluated and transferred to Yeast extract- malt extract agar (ISP-2) plates (Yeast extract, 2g; Malt extract, 10g and Dextrose, $10 \mathrm{~g}$ ) to check the purity and maintained by periodical sub culturing.

\section{Semi-quantitative screening of Enzyme Activities:-}

The Streptomyces strains were initially screened for detecting exo - $\beta$-1,4-glucanase activity on synthetic medium containing Avicel (Sigma, USA) as the sole carbon source. The composition of medium was as follows: Avicel,1\%; yeast extract, $0.1 \%$; Peptone, $0.1 \%$; $\mathrm{KH}_{2} \mathrm{PO}_{4}, 0.1 \%$ and $\mathrm{MgSO}_{4} .7 \mathrm{H}_{2} \mathrm{O}, 0.05 \%$. The strains were spot inoculated on Avicel agar plates and incubated for 5 days at $28^{\circ} \mathrm{C}$. After incubation $0.1 \%$ congo red dye was spreaded over the plates and washed with 1 mol. $\mathrm{L}^{-1} \mathrm{NaCl}$, used as a destaining solution. Exo- $\beta$-1,4-glucanase production was indicated by the presence of a pale halo around the colonies. A semi quantitative approach was used for measuring the enzyme activity that is the enzymatic index (EI) of strains were calculated using the expression.

$$
E I=\frac{\text { Diameterofhydrolysiszone }(\emptyset h)}{\text { Diameterof colony }(\emptyset c)}
$$

The endo- $\beta$-1,3-glucanase activity was screened using AZCL- Pachyman (Megazyme, USA) agar plates with medium composition of AZCL- Pachyman, 0.2\%; Yeast extract, 0.1\%; Peptone,0.1\%; $\mathrm{KH}_{2} \mathrm{PO}_{4}, 0.1 \%$ and $\mathrm{MgSO}_{4} .7 \mathrm{H}_{2} \mathrm{O}, 0.05 \%$ by spot inoculation and incubated 5 days at $28^{\circ} \mathrm{C}$. Presence of blue zones around the colonies were indicated as enzyme activity by potential strains. The EI values were calculated by measuring diameters of blue halo and colony.

Protease production of Streptomyces isolates were screened on skimmed milk agar containing Casein enzyme hydrolysate, 5\%; Yeast extract, 2.5 and Glucose, 1\%. The plates were spot inoculated and incubated at room temperature for 5 days at $28^{\circ} \mathrm{C}$. Positive isolates were detected by the appearance of clear halos around the colonies. The enzymatic index (EI) of strains were calculated.

\section{Quantitative Enzyme Assays:-}

The exo- $\beta$-1,4-glucanase assay was carried out by measuring reducing sugars by DNS method (Miller, 1959) liberated from $0.5 \%(\mathrm{w} / \mathrm{v})$ Avicel which is dissolved in $100 \mathrm{mM}$ sodium- acetate buffer (pH 5.0) was used as substrate. $0.5 \mathrm{ml}$ of filtrate was added to $1 \mathrm{ml}$ of $0.5 \%$ Avicel and incubated at $30^{\circ} \mathrm{C}$ for $1 \mathrm{hr}$ in shaker incubator. $2 \mathrm{ml}$ of DNS reagent was added and incubated in boiling water bath for 5 minutes. After adding $0.5 \mathrm{ml} 40 \%$ Rochelle salt the absorbance was taken at $540 \mathrm{~nm}$ wavelength using visible spectrophotometer. One unit of exo- $\beta$-1,4-glucanase activity $\left(\mathrm{U} \mathrm{mL}^{-1}\right)$ was defined as the amount of enzyme that released $1 \mu \mathrm{mol}$ of reducing sugars as glucose equivalents from one $\mathrm{ml}$ of Avicel per minute under given assay conditions (Oliveira et al., 2014).

Endo- $\beta$-1,3-glucanase activity was estimated by measuring the release of reducing sugars in a reaction mixture of $0.5 \mathrm{ml}$ of enzyme and $1 \mathrm{ml} 0.2 \%$ (w/v) CM- curdlan (Megazyme, USA) in 100mM sodium- acetate buffer (pH 5.0) incubated at $30^{\circ} \mathrm{C}$ for $1 \mathrm{hr}$ and the remaining procedures were similar as exo- $\beta$-1,4-glucanase assay. Enzyme activity was expressed by $1 \mu \mathrm{mol}$ of reducing sugar released per minute under standard assay conditions.

The protease enzyme activity was determined by a modified method of Rupali (2015). Casein is used as substrate for determination of protease activity. $1 \%(\mathrm{w} / \mathrm{v})$ of casein in $50 \mathrm{mM}$ phosphate buffer $(\mathrm{pH} 7.0)$ was used as substrate and incubated with $0.5 \mathrm{ml}$ enzyme solution at $37^{\circ} \mathrm{C}$ for 30 minutes. The reaction was stopped by adding $2 \mathrm{ml}$ of $100 \mathrm{mM}$ trichloroacetic acid. After centrifugation at $7000 \mathrm{rpm}$ for 15 minutes, $0.5 \mathrm{ml}$ supernatant was taken and added $2 \mathrm{ml}$ $0.5 \mathrm{M} \mathrm{Na}_{2} \mathrm{CO}_{3}$ and $0.5 \mathrm{ml}$ diluted Folin phenol reagent, then kept it in incubation under dark conditions for 30 minutes. The absorbance was read at $660 \mathrm{~nm}$. One unit of protease activity is defined as the amount of enzyme that liberated $1 \mu$ mole of tyrosine per $\mathrm{ml}$ per minute under standard assay conditions.

Determination of Soil pH and Electrical Conductivity:-

Soil $\mathrm{pH}$ and electrical conductivity (EC) were determined in 1: 3.0 soil/water ratio by a combination glass electrode HI98129, Hanna Instruments. 


\section{Determination of Soil Organic Carbon and Soil Organic Matter:-}

The soil organic carbon (SOC) content was estimated by dichromate oxidation method in which the oxidation of $\mathrm{K}_{2} \mathrm{Cr}_{2} \mathrm{O}_{7}$ in a concentrated $\mathrm{H}_{2} \mathrm{SO}_{4}$ medium and the excess dichromate was measured using $\left(\mathrm{NH}_{4}\right)_{2} \mathrm{Fe}\left(\mathrm{SO}_{4}\right)_{2}$ (Yeomans and Bremner, 1989). Soil organic matter (SOM) were determined according to Pribyl, 2010.

\section{Statistical analysis:-}

All part of experiments in this study were performed in triplicate. Data were presented as mean $\pm \mathrm{SD}$. The $\mathrm{p}$ values $<0.05$ were considered statistically significant. To determine the correlations between the parameters linear regression analysis (Pearson method) was performed.

\section{Results and Discussion:-}

Number of Streptomyces Isolates:-

Morphologically different 95 Streptomyces strains were isolated from 21 locations of three different land areas viz. highland, midland and low land. The number of strains showed a decreasing trend from highland to lowland (Figure 2). Soils from natural ecosystem were observed higher microbial content when compared to agricultural ecosystems. Highland areas in Kerala mainly Western Ghats regions represents high levels of biological diversity, less unexplored and mostly covers with protected land mass. Due to the lack of anthropogenic activities, number of Streptomyces isolates are significantly high in these areas when compared with other two geographical landmasses such as midland and lowland areas. Taxonomically diverse Streptomyces species are found in forest soils, they are involved in recalcitrant biopolymer degradation. This leads to the prominence of Streptomyces in forest nutrient turnover (Bontemps et al., 2013). According to Zhou et al., 2016, rich plant biomass in paddy field expects high number of hydrolase producing organisms, however gets only very small fraction of isolates from the area. Application of the high level of mineral fertilization along with chemical weed control agents predominantly detriments the biological activity of the soil because of higher loss of micro-organisms due to rigorous chemical plant protection (Bielińska and Pranagal 2007). The number of isolates showing a decreasing trend from highland to midland to lowland. This study, clearly reveals the relation between ecological variations and Streptomyces population, hence number of Streptomyces isolates can be considered as a delicate indicator of environmental changes.

\section{Screening of Enzyme Activities:-}

All the 95 isolates were evaluated for semi-quantitative exo- $\beta-1,4$-glucanase, endo- $\beta$-1,3-glucanase and protease production. From the total 95 isolates 85 strains were shown exo- $\beta-1,4$-glucanase activity (equivalent to $89 \%$ of the strains evaluated) 60 shown endo- $\beta$-1,3-glucanase activity (equivalent to $63 \%$ of the strains evaluated) and only 61 shown protease activity (equivalent to $64 \%$ of the strains evaluated). This is based on the observation of growth and measurement of hydrolysis halo, used for calculation of the enzymatic index (EI). The pale halo around the exo- $\beta$ 1,4-glucanolytic colonies corresponds to the zone of Avicel (specific substrate for exo- $\beta$-1,4-glucanase) degradation (Figure 3a), blue colouration around endo- $\beta-1,3$-glucanolytic colonies indicating the zone of Pachyman (specific substrate for endo- $\beta$-1,3-glucanase) degradation (Figure $3 \mathrm{~b}$ ) and appearance of clear zone around the colonies surrounded by white color background indicated the presence of protease activity, corresponds to the zone of protein degradation (Figure 3c).

Strains from different land areas were given significantly wide ranges of EI. Exo- $\beta-1,4$-glucanase activity was more prominent in high, mid and lowland isolates. In highland isolates, EI of exo- $\beta-1,4$-glucanase, endo- $\beta$-1,3-glucanase and protease were shown in between 2.17 to $6.03,2.0$ to 8.53 and 1.03 to 3.03 respectively (Table 2). Midland isolate EI values were ranged from 1.80 to $7.27,1.8$ to 5.0 and 1.20 to 3.13 for exo- $\beta$-1,4-glucanase, endo- $\beta-1,3$ glucanase and protease respectively (Table 3 ). In lowland soil isolates the EI values of exo- $\beta$-1,4-glucanase was in between 1.50 to 4.20 , endo- $\beta-1,3$-glucanase in between 2.30 to 3.63 and 1.40 to 3.03 for protease (Table 4 ). 
Table 1:- Location, elevation and descriptions of soil sampling areas.

\begin{tabular}{|c|c|c|c|c|}
\hline Area & Location & Elevation & Description & Land use type \\
\hline \multicolumn{5}{|l|}{ Highland } \\
\hline Chinnar & $\begin{array}{c}10^{\circ} 18^{\prime} 20.999^{\prime} \mathrm{N} \text { latitude } \\
77^{\circ} 12^{\prime} 19.36^{\prime \prime} \mathrm{E} \text { longitude }\end{array}$ & $1,005 \mathrm{~m}$ & $\begin{array}{l}\text { Lower mountain } \\
\text { forest }\end{array}$ & $\begin{array}{l}\text { Protected area with } \\
\text { natural vegetation }\end{array}$ \\
\hline Munnar & $\begin{array}{l}10^{\circ} 05^{\prime} 21.29^{\prime} \mathrm{N} \text { latitude } \\
77^{\circ} 03^{\prime} 27.16^{\prime \prime} \mathrm{E} \text { longitude }\end{array}$ & $1,477 \mathrm{~m}$ & Hill station & Natural vegetation \\
\hline Marayur & $\begin{array}{l}10^{\circ} 15^{\prime} 11.91 \text { 'N latitude } \\
77^{\circ} 09 ' 31.20^{\prime \prime} \mathrm{E} \text { longitude }\end{array}$ & $949 \mathrm{~m}$ & $\begin{array}{c}\text { Natural sandal } \\
\text { wood forest }\end{array}$ & Natural vegetation \\
\hline Anaimalai & $\begin{array}{l}10^{\circ} 10^{\prime} 02.62^{\prime} \mathrm{N} \text { latitude } \\
77^{\circ} 03 ' 51.67^{\prime \prime} \mathrm{E} \text { longitude }\end{array}$ & $1,577 \mathrm{~m}$ & Mountain & Natural vegetation \\
\hline Nelliyampathy & $\begin{array}{c}10^{\circ} 32^{\prime} 00.73^{\prime \prime} \mathrm{N} \text { latitude } \\
76^{\circ} 41^{\prime} 35.76^{\prime \prime} \mathrm{E} \text { longitude }\end{array}$ & $999 \mathrm{~m}$ & Hill range & Natural vegetation \\
\hline Agasthyakoodam & $\begin{array}{c}8^{\circ} 37^{\prime} 29.47^{\prime \prime} \mathrm{N} \text { latitude } \\
77^{\circ} 14^{\prime} 08.21^{\prime \prime} \mathrm{E} \text { longitude }\end{array}$ & $1,238 \mathrm{~m}$ & Hill range & Natural vegetation \\
\hline Wayanad & $\begin{array}{c}11^{\circ} 36^{\prime} 49.41^{\prime \prime} \mathrm{N} \text { latitude } \\
76^{\circ} 06^{\prime} 05.22^{\prime \prime} \mathrm{E} \text { longitude }\end{array}$ & $957 \mathrm{~m}$ & Mountain forest & Natural vegetation \\
\hline \multicolumn{5}{|l|}{ Midland } \\
\hline Bonacaud & $\begin{array}{c}8^{\circ} 40^{\prime} 47.52^{\prime \prime} \mathrm{N} \text { latitude } \\
77^{\circ} 10^{\prime} 00.644^{\prime \prime} \text { longitude }\end{array}$ & $336 \mathrm{~m}$ & Protected forest & Natural vegetation \\
\hline Palode, & $\begin{array}{c}8^{\circ} 44^{\prime} 32.04 \text { 'N latitude } \\
77^{\circ} 01 \text { '34.67''E longitude }\end{array}$ & $115 \mathrm{~m}$ & Reserve forest & Plantation \\
\hline Kallar & $\begin{array}{c}8^{\circ} 42^{\prime} 36.39 \text { 'N latitude } \\
77^{\circ} 07^{\prime} 16.01^{\prime \prime} \mathrm{E} \text { longitude }\end{array}$ & $113 \mathrm{~m}$ & Natural forest & Natural vegetation \\
\hline Neryamangalam & $\begin{array}{c}10^{\circ} 02 ' 57.52 \text { 'N latitude } \\
76^{\circ} 46^{\prime} 19.82^{\prime \prime} \mathrm{E} \text { longitude }\end{array}$ & $110 \mathrm{~m}$ & Natural forest & Natural vegetation \\
\hline Kothamangalam & $\begin{array}{c}10^{\circ} 03 ' 50.51^{\prime \prime} \mathrm{N} \text { latitude } \\
76^{\circ} 39^{\prime} 07.24^{\prime \prime} \mathrm{E} \text { longitude }\end{array}$ & $55 \mathrm{~m}$ & Cultivated area & Agricultural vegetation \\
\hline Ottapalam & $\begin{array}{c}10^{\circ} 46^{\prime} 32.844^{\prime \prime} \mathrm{N} \text { latitude } \\
76^{\circ} 21^{\prime} 34.19^{\prime \prime} \mathrm{E} \text { longitude }\end{array}$ & $63 \mathrm{~m}$ & Cultivated area & Agricultural vegetation \\
\hline Thaliparamba & $\begin{array}{c}12^{\circ} 02 ' 20.71 \text { 'N latitude } \\
75^{\circ} 21^{\prime} 59.93 \text { 'E longitude }\end{array}$ & $64 \mathrm{~m}$ & Cultivated area & Agricultural vegetation \\
\hline \multicolumn{5}{|l|}{ Lowland } \\
\hline Kainakkari & $\begin{array}{c}9^{\circ} 30^{\prime} 44.53 \text { 'N latitude } \\
76^{\circ} 233^{\prime} 38.81^{\prime \prime} \mathrm{E} \text { longitude }\end{array}$ & $1 \mathrm{~m}$ & Paddy field & Agricultural vegetation \\
\hline Puthupalli & $\begin{array}{c}9^{\circ} 33^{\prime} 22.28^{\prime \prime} \mathrm{N} \text { latitude } \\
76^{\circ} 33^{\prime} 37.64^{\prime \prime} \mathrm{E} \text { longitude }\end{array}$ & $3 \mathrm{~m}$ & Paddy field & Agricultural vegetation \\
\hline Mankombu & $\begin{array}{c}9^{\circ} 26^{\prime} 08.77^{\prime \prime} \mathrm{N} \text { latitude } \\
76^{\circ} 24^{\prime} 30.81^{\prime \prime} \mathrm{E} \text { longitude }\end{array}$ & $1 \mathrm{~m}$ & Paddy field & Agricultural vegetation \\
\hline Changanasseri & $\begin{array}{c}10^{\circ} 18^{\prime} 20.999^{\prime} \mathrm{N} \text { latitude } \\
77^{\circ} 12^{\prime} 19.36^{\prime \prime} \mathrm{E} \text { longitude }\end{array}$ & $1 \mathrm{~m}$ & Paddy field & Agricultural vegetation \\
\hline Mavelikkara & $\begin{array}{c}9^{\circ} 15^{\prime} 38.79^{\prime \prime} \mathrm{N} \text { latitude } \\
76^{\circ} 30^{\prime} 44.35^{\prime} \text { 'E longitude }\end{array}$ & $2 \mathrm{~m}$ & Paddy field & Agricultural vegetation \\
\hline Nedungad & $\begin{array}{c}10^{\circ} 04 ' 36.91 ' \mathrm{~N} \text { latitude } \\
76^{\circ} 13 \text { '02.35'” longitude }\end{array}$ & $2 \mathrm{~m}$ & Paddy field & Agricultural vegetation \\
\hline Thiruvalla & $\begin{array}{c}9^{\circ} 24^{\prime} 37.32^{\prime \prime} \mathrm{N} \text { latitude } \\
76^{\circ} 32^{\prime} 01.36^{\prime \prime} \text { E longitude }\end{array}$ & $1 \mathrm{~m}$ & Paddy field & Agricultural vegetation \\
\hline
\end{tabular}


Table 2:- Primary screening of exo- $\beta$-1,4-glucanase, endo- $\beta$-1,3-glucanase and protease in highland isolates. Data are expressed as mean \pm standard deviation (SD) of three replicates. Values are statistically significant at $\mathrm{P}<0.05$

\begin{tabular}{|c|c|c|c|}
\hline & Exo- $\beta$-1,4- glucanase & Endo- $\beta-1,3$ - glucanase & Protease \\
\hline Strain & Mean EI \pm SD & Mean EI \pm SD & Mean EI \pm SD \\
\hline ch1 & $4.10 \pm 0.089$ & $3.60 \pm 0.200$ & NA \\
\hline $\operatorname{ch} 3$ & $4.30 \pm 0.155$ & $2.50 \pm 0.300$ & $1.63 \pm 0.153$ \\
\hline ch5 & $3.73 \pm 0.137$ & $2.13 \pm 0.252$ & $1.83 \pm 0.321$ \\
\hline $\operatorname{ch} 7$ & $4.13 \pm 0.137$ & $3.00 \pm 0.300$ & NA \\
\hline ch8 & $3.80 \pm 0.089$ & NA & NA \\
\hline $\operatorname{ch} 15$ & $4.13 \pm 0.052$ & NA & NA \\
\hline ch16 & $4.20 \pm 0.089$ & $\mathrm{NA}$ & NA \\
\hline $\operatorname{ch} 18$ & $4.10 \pm 0.322$ & NA & $1.53 \pm 0.351$ \\
\hline $\operatorname{ch} 20$ & $4.00 \pm 0.089$ & $2.80 \pm 0.100$ & $1.80 \pm 0.100$ \\
\hline $\operatorname{ch} 21$ & $4.20 \pm 0.179$ & $3.40 \pm 0.458$ & $1.50 \pm 0.265$ \\
\hline $\operatorname{ch} 22$ & $4.50 \pm 0.089$ & $8.53 \pm 0.058$ & $1.30 \pm 0.100$ \\
\hline $\operatorname{ch} 23$ & $4.00 \pm 0.089$ & $3.00 \pm 0.346$ & NA \\
\hline al6 & $3.30 \pm 0.089$ & NA & NA \\
\hline al9 & $4.03 \pm 0.052$ & $\mathrm{NA}$ & NA \\
\hline al10 & $3.87 \pm 0.052$ & NA & $1.20 \pm 0.200$ \\
\hline al13 & $2.73 \pm 0.137$ & $6.90 \pm 0.100$ & $1.50 \pm 0.200$ \\
\hline al14 & $2.30 \pm 0.155$ & $4.60 \pm 0.458$ & NA \\
\hline al19 & $6.03 \pm 0.137$ & $3.10 \pm 0.265$ & $1.30 \pm 0.265$ \\
\hline al22 & $2.50 \pm 0.089$ & $2.00 \pm 0.300$ & $1.03 \pm 0.208$ \\
\hline $\mathrm{mn} 1$ & $3.83 \pm 0.137$ & $3.10 \pm 0.200$ & $2.07 \pm 0.115$ \\
\hline $\mathrm{mn} 2$ & $4.07 \pm 0.207$ & $2.10 \pm 0.200$ & $1.90 \pm 0.100$ \\
\hline $\mathrm{mn} 3$ & $3.13 \pm 0.137$ & $3.80 \pm 0.265$ & NA \\
\hline mn5 & $4.67 \pm 0.186$ & $3.80 \pm 0.265$ & $1.40 \pm 0.100$ \\
\hline mn6 & $3.47 \pm 0.137$ & $2.60 \pm 0.300$ & $3.00 \pm 0.173$ \\
\hline $\mathrm{mn} 7$ & $3.80 \pm 0.089$ & $3.80 \pm 0.200$ & $2.00 \pm 0.300$ \\
\hline $\mathrm{mn} 8$ & $4.13 \pm 0.137$ & $4.10 \pm 0.361$ & $3.03 \pm 0.252$ \\
\hline mn9 & $4.27 \pm 0.137$ & $2.75 \pm 0.466$ & $1.90 \pm 0.200$ \\
\hline $\mathrm{mn} 10$ & $3.40 \pm 0.089$ & $3.10 \pm 0.265$ & $1.43 \pm 0.153$ \\
\hline $\mathrm{mn} 11$ & $3.53 \pm 0.137$ & $2.50 \pm 0.265$ & $2.20 \pm 0.200$ \\
\hline mn12 & $4.13 \pm 0.137$ & NA & $2.00 \pm 0.200$ \\
\hline $\mathrm{km} 2$ & $3.40 \pm 0.089$ & $4.10 \pm 0.173$ & $1.23 \pm 0.153$ \\
\hline $\mathrm{mr} 1$ & $4.07 \pm 0.137$ & $2.57 \pm 0.306$ & $2.57 \pm 0.153$ \\
\hline $\mathrm{mr} 3$ & $4.77 \pm 0.137$ & $3.10 \pm 0.100$ & $3.03 \pm 0.252$ \\
\hline mr8 & $5.03 \pm 0.052$ & $2.90 \pm 0.200$ & $3.03 \pm 0.153$ \\
\hline mr9 & $4.67 \pm 0.186$ & NA & $1.90 \pm 0.100$ \\
\hline $\mathrm{mr} 7$ & $2.17 \pm 0.137$ & $4.80 \pm 0.300$ & $2.03 \pm 0.306$ \\
\hline $\mathrm{mr} 2$ & $3.57 \pm 0.052$ & $4.60 \pm 0.100$ & $1.30 \pm 0.265$ \\
\hline $\operatorname{mr} 17$ & $5.03 \pm 0.137$ & $3.10 \pm 0.265$ & NA \\
\hline $\operatorname{mr} 18$ & $3.47 \pm 0.052$ & NA & NA \\
\hline mr19 & $3.75 \pm 0.089$ & $2.50 \pm 0.265$ & NA \\
\hline ag21 & $4.03 \pm 0.186$ & NA & $2.50 \pm 0.173$ \\
\hline ag28 & $4.10 \pm 0.089$ & $2.50 \pm 0.300$ & $1.93 \pm 0.115$ \\
\hline nmp6 & NA & NA & NA \\
\hline nmp5-3 & NA & $3.30 \pm 0.265$ & $3.03 \pm 0.306$ \\
\hline amla5 & $4.40 \pm 0.089$ & $3.90 \pm 0.100$ & NA \\
\hline amla31 & $3.40 \pm 0.155$ & $2.50 \pm 0.265$ & NA \\
\hline ala8III & $4.10 \pm 0.473$ & $4.30 \pm 0.265$ & $2.10 \pm 0.200$ \\
\hline a6-2 & $3.80 \pm 0.237$ & $3.33 \pm 0.416$ & $1.50 \pm 0.100$ \\
\hline wy2 & $3.40 \pm 0.358$ & $3.40 \pm 0.173$ & $1.23 \pm 0.153$ \\
\hline wy5 & $3.40 \pm 0.358$ & $3.70 \pm 0.200$ & $1.93 \pm 0.058$ \\
\hline wy8 & $3.40 \pm 0.358$ & $4.20 \pm 0.361$ & $1.83 \pm 0.153$ \\
\hline wy9 & $4.50 \pm 0.322$ & $3.60 \pm 0.265$ & $1.50 \pm 0.173$ \\
\hline s40a5 & $3.50 \pm 0.179$ & $3.00 \pm 0.200$ & NA \\
\hline
\end{tabular}


Table 3:- Primary screening of exo- $\beta$-1,4-glucanase, endo- $\beta$-1,3-glucanase and protease in midland isolates. Data are expressed as mean \pm standard deviation (SD) of three replicates. Values are statistically significant at $\mathrm{P}<0.05$

\begin{tabular}{|c|c|c|c|}
\hline & Exo- $\beta-1,4$ - glucanase & Endo- $\beta-1,3$ - glucanase & Protease \\
\hline Strain & Mean EI \pm SD & Mean EI \pm SD & Mean EI \pm SD \\
\hline nr2 & $4.63 \pm 0.137$ & $4.60 \pm 0.346$ & $1.50 \pm 0.200$ \\
\hline nr3 & $7.27 \pm 0.225$ & NA & NA \\
\hline nr1 & $4.63 \pm 0.052$ & $3.50 \pm 0.361$ & $1.53 \pm 0.208$ \\
\hline nr22 & $3.37 \pm 0.137$ & NA & NA \\
\hline nr23 & $3.90 \pm 0.089$ & $3.30 \pm 0.300$ & $2.03 \pm 0.153$ \\
\hline nr24 & $2.97 \pm 0.186$ & NA & $1.30 \pm 0.265$ \\
\hline pa1 & $4.00 \pm 0.179$ & $4.30 \pm 0.200$ & $1.53 \pm 0.252$ \\
\hline pa4 & $3.90 \pm 0.089$ & $5.00 \pm 0.600$ & $1.40 \pm 0.200$ \\
\hline pa7 & $3.07 \pm 0.052$ & NA & NA \\
\hline kr6 & $4.07 \pm 0.103$ & $3.30 \pm 0.265$ & $1.20 \pm 0.265$ \\
\hline kr7 & $4.47 \pm 0.052$ & $2.80 \pm 0.100$ & $1.40 \pm 0.200$ \\
\hline ko1 & $4.80 \pm 0.179$ & $3.40 \pm 0.361$ & $1.73 \pm 0.208$ \\
\hline ko6 & NA & NA & NA \\
\hline ko8 & $5.10 \pm 0.089$ & $3.20 \pm 0.361$ & $3.13 \pm 0.321$ \\
\hline ko9 & $4.50 \pm 0.089$ & $5.00 \pm 0.600$ & $1.70 \pm 0.200$ \\
\hline ot5 & $4.12 \pm 0.207$ & $3.10 \pm 0.100$ & NA \\
\hline ot7 & $4.20 \pm 0.155$ & $3.50 \pm 0.300$ & $\mathrm{NA}$ \\
\hline ot10 & $\mathrm{NA}$ & $3.90 \pm 0.265$ & $\mathrm{NA}$ \\
\hline ot11 & $\mathrm{NA}$ & $\mathrm{NA}$ & $2.10 \pm 0.200$ \\
\hline th1 & $4.33 \pm 0.273$ & $3.30 \pm 0.436$ & $2.03 \pm 0.153$ \\
\hline th2 & $\mathrm{NA}$ & $2.50 \pm 0.300$ & $2.30 \pm 0.265$ \\
\hline th5 & $3.40 \pm 0.155$ & $3.50 \pm 0.300$ & $\mathrm{NA}$ \\
\hline b1 & $\mathrm{NA}$ & NA & $\mathrm{NA}$ \\
\hline b2 & $1.80 \pm 0.237$ & $1.80 \pm 0.300$ & $1.40 \pm 0.100$ \\
\hline b3 & $\mathrm{NA}$ & NA & $1.30 \pm 0.265$ \\
\hline $\mathrm{b} 4$ & NA & NA & NA \\
\hline b5 & $\mathrm{NA}$ & $4.60 \pm 0.346$ & $1.50 \pm 0.200$ \\
\hline
\end{tabular}

Table 4:- Primary screening of exo- $\beta$-1,4-glucanase, endo- $\beta-1,3$-glucanase and protease in lowland isolates. Data are expressed as mean \pm standard deviation (SD) of three replicates. Values are statistically significant at $\mathrm{P}<0.05$

\begin{tabular}{|c|c|c|c|}
\hline & Exo- $\beta-1,4-$ glucanase & Endo- $\beta-1,3-$ glucanase & Protease \\
\hline Strain & Mean EI \pm SD & Mean EI \pm SD & Nean EI \pm SD \\
\hline pl1 & $3.40 \pm 0.082$ & NA & $2.33 \pm 0.153$ \\
\hline p12 & $3.40 \pm 0.216$ & $3.03 \pm 0.153$ & NA \\
\hline p13 & $1.50 \pm 0.163$ & $2.77 \pm 0.252$ & NA \\
\hline mk5 & $3.13 \pm 0.125$ & $3.10 \pm 0.200$ & $1.47 \pm 0.416$ \\
\hline mk6 & $3.60 \pm 0.245$ & $2.83 \pm 0.351$ & $2.50 \pm 0.200$ \\
\hline cs2 & $2.30 \pm 0.216$ & $3.60 \pm 0.300$ & NA \\
\hline cs3 & $3.30 \pm 0.283$ & NA & $2.80 \pm 0.200$ \\
\hline mv1 & $4.00 \pm 0.163$ & $3.63 \pm 0.351$ & $1.70 \pm 0.200$ \\
\hline mv2 & $2.30 \pm 0.216$ & $2.30 \pm 0.300$ & NA \\
\hline mv3 & $3.60 \pm 0.082$ & $2.87 \pm 0.321$ & NA \\
\hline nd5 & $3.60 \pm 0.141$ & $2.90 \pm 0.200$ & $1.40 \pm 0.265$ \\
\hline th1 & $2.80 \pm 0.163$ & NA & $3.03 \pm 0.252$ \\
\hline th2 & $1.70 \pm 0.163$ & NA & NA \\
\hline ki2 & $1.70 \pm 0.082$ & $2.90 \pm 0.100$ & \\
\hline ki3 & $4.20 \pm 0.163$ & $3.30 \pm 0.265$ & \\
\hline
\end{tabular}




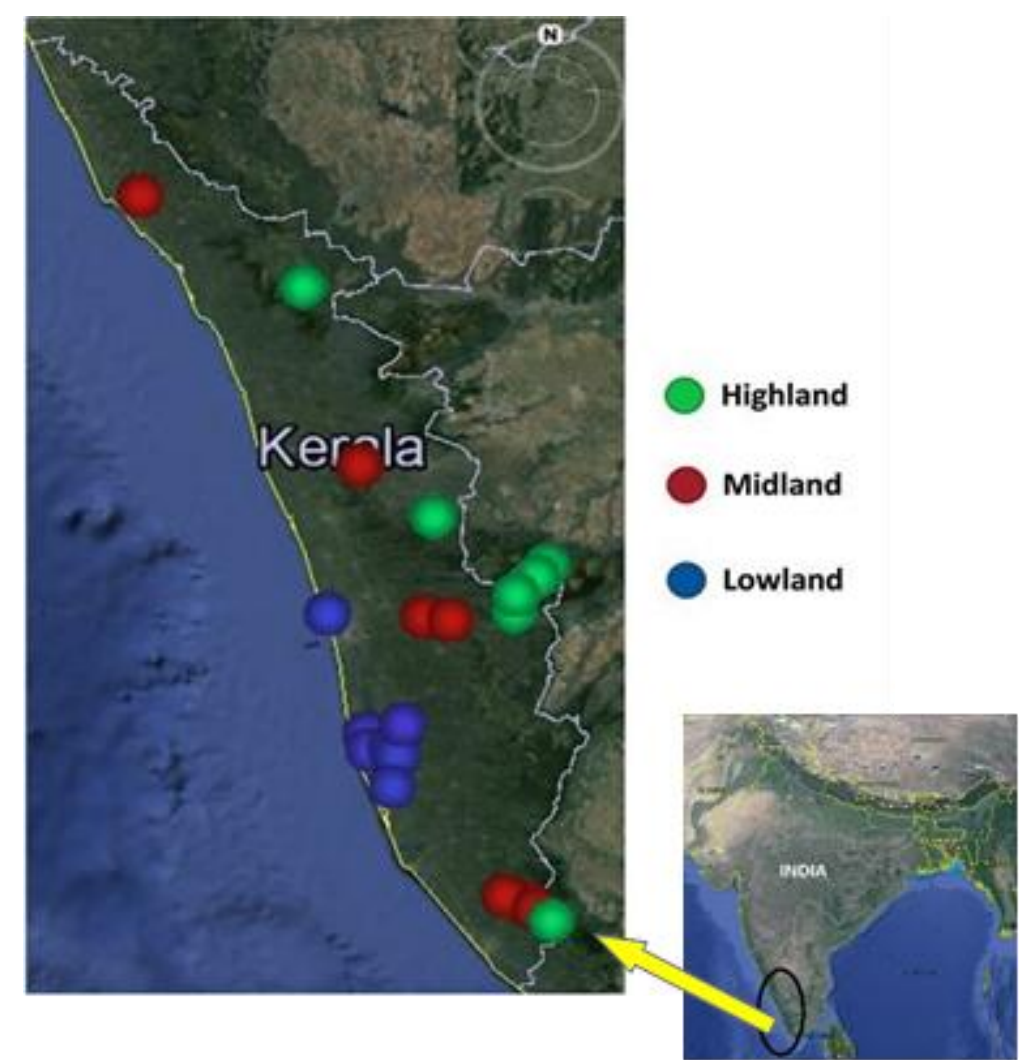

Figure 1:- Map showing soil sample collection sites.

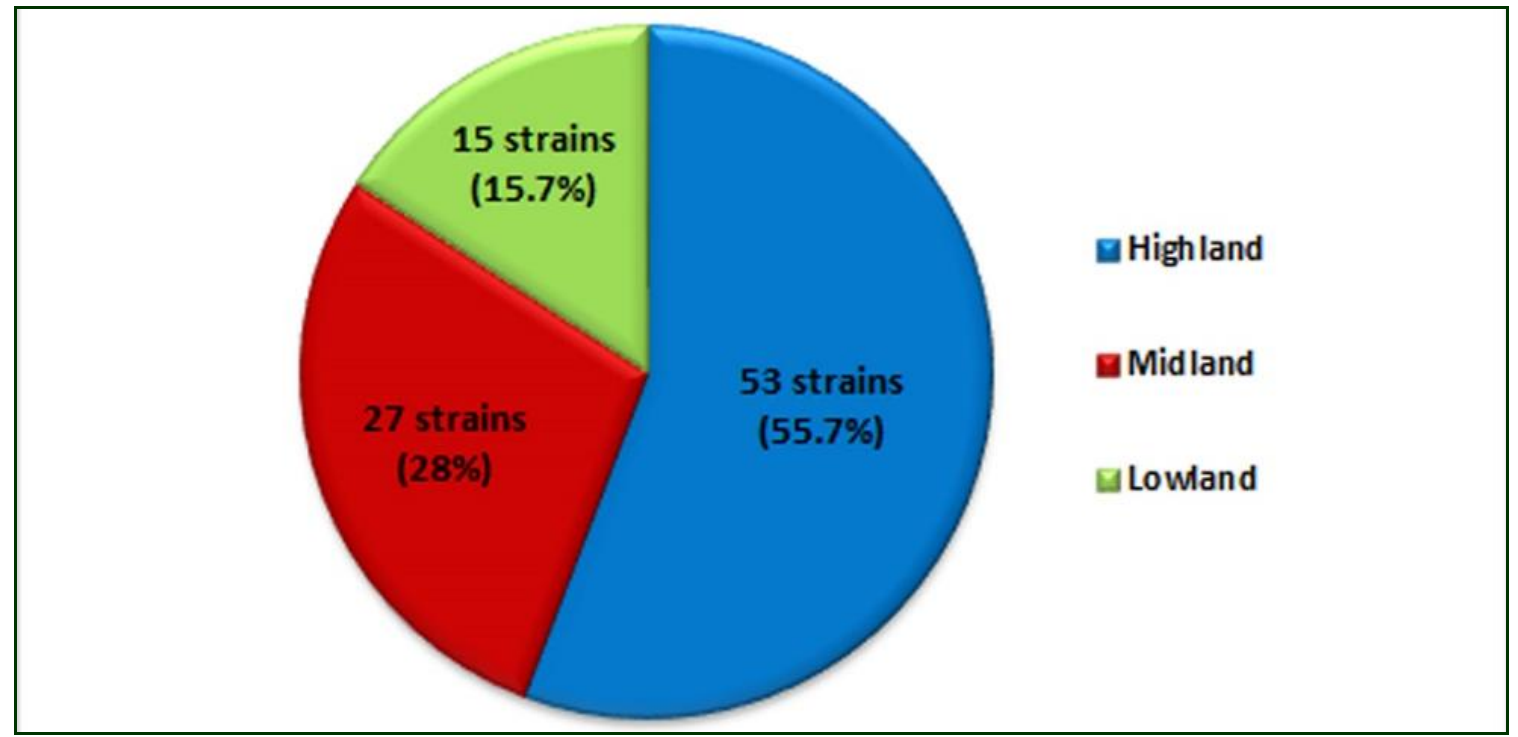

Fig. 2:- Pie chart showing the percentage profile of Streptomyces isolates from highland, midland and lowland areas. 

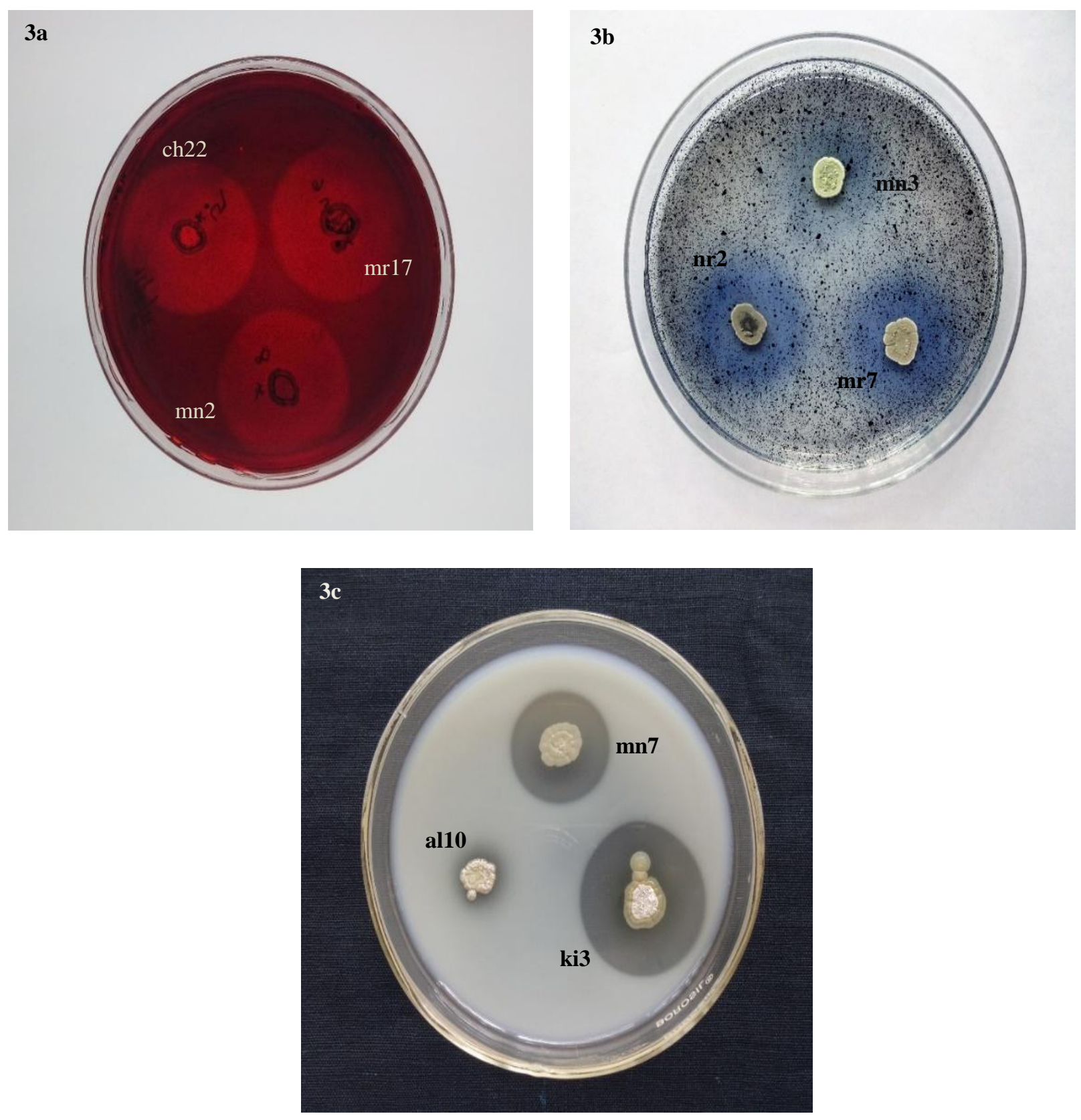

Figure 3:- Semi-quantitative screening plates of exo- $\beta-1,4$-glucanase (3a), endo- $\beta-1,3$-glucanase (3b) and proteases $(3 \mathrm{c})$. 

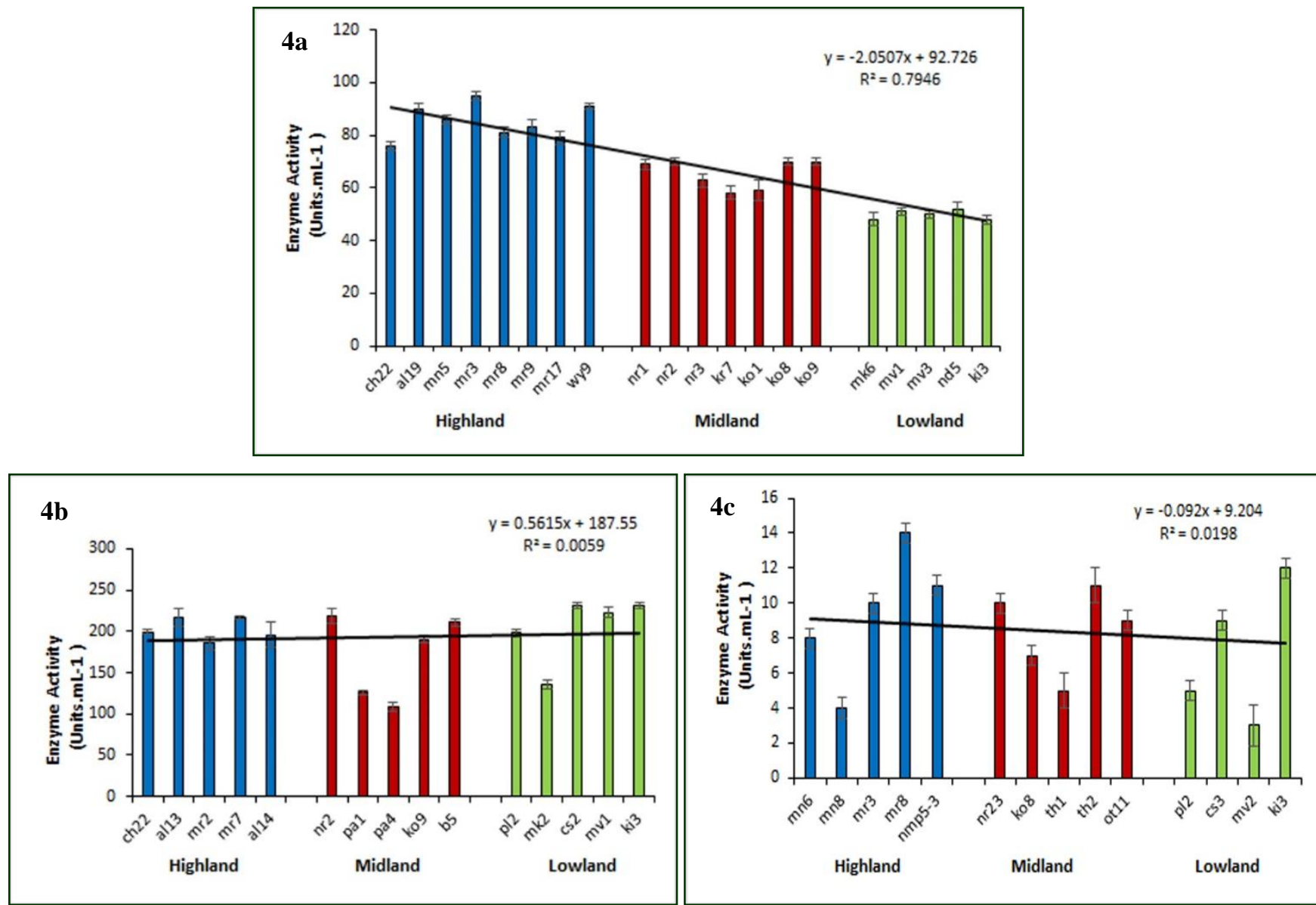

Figure 4:- Comparison of exo- $\beta$-1,4-glucanase (4a), endo- $\beta$-1,3-glucanase (4b) and protease (4c) activities in highland, midland and lowland. Datas are expressed as mean \pm standard deviation (SD) of three replicates. Regression equation and line of fit shown. Values are statistically significant at $\mathrm{P}<0.05$.

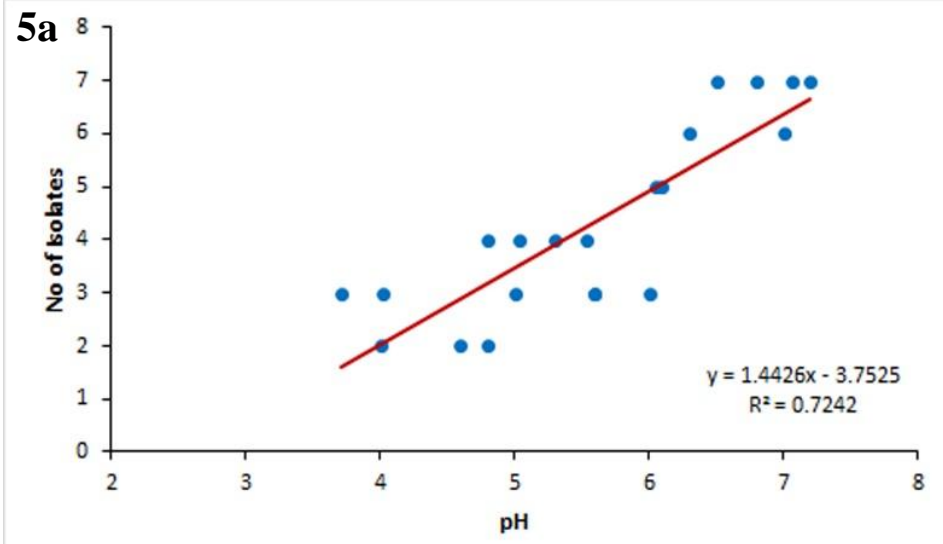




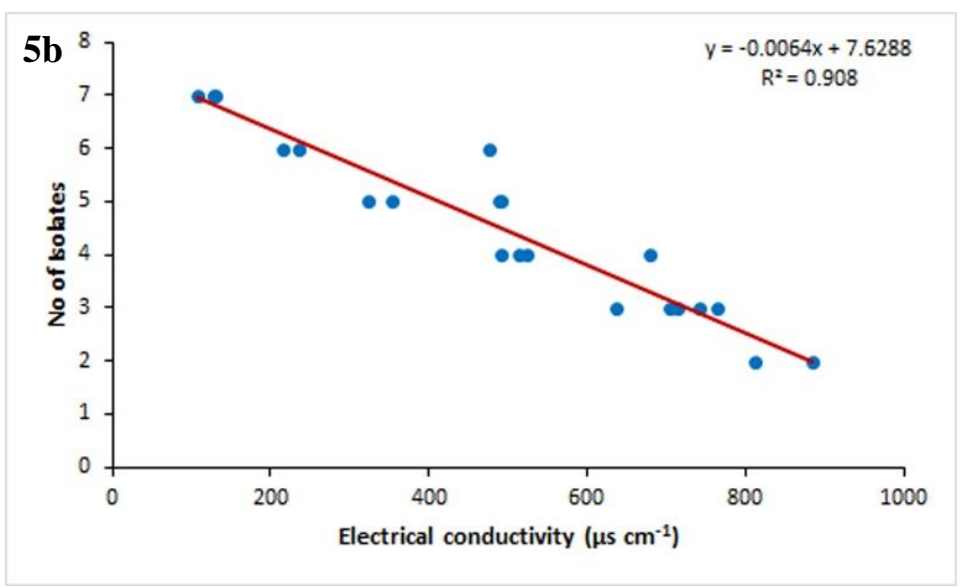

Figure 5:- Linear regression between numbers of Streptomyces isolates and soil $\mathrm{P}^{\mathrm{H}}$ (5a); Electrical conductivity (5b)

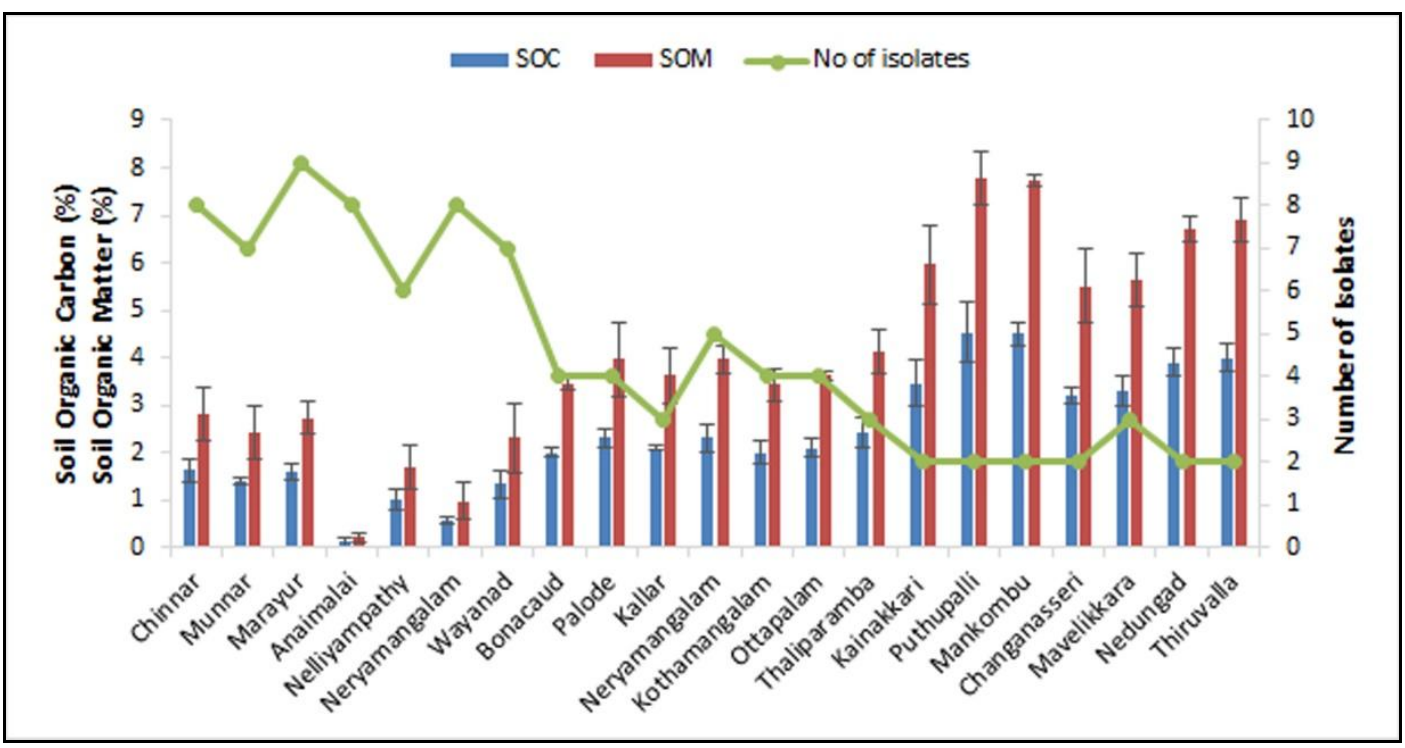

Figure 6:- Relationship of soil organic carbon (SOC) and soil organic matter (SOM) with number of isolates. Datas are expressed as mean \pm standard deviation (SD) of three replicates. Values are statistically significant at $\mathrm{P}<0.05$.

\section{Quantitative Enzyme Assays:-}

As a result of semi-quantitative primary screening, strains with high EI values were considered as potent enzyme producers and were selected for quantitative enzyme assays. 8 isolates from highland areas and 7 isolates from midland areas, both expressed EI values in and above 4.5 and 5 isolates from low land area with EI values in and above 3.5 were selected for exo- $\beta$-1,4-glucanase assay. Endo- $\beta$-1,3-glucanase assay was carried out with highland strains showed EI values in and above 4.5, midland strains with EI values in and above 4.0 and lowland strains with EI values in and above 3.0. Protease quantitative assay was done with selected isolates from highland with EI values in and above 3.0, midland and lowland with EI values in and above 2.0.

The quantitative assay clearly revealed that activity of exo- $\beta-1,4$-glucanase in highland was ranged between 95 to 76

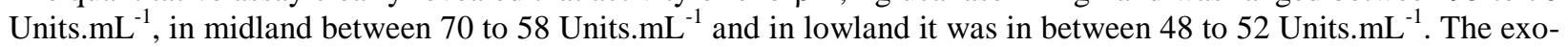
$\beta$-1,4-glucanase activity was recorded highest in highland areas and gradually decreased in low land areas (Figure 4a). Here the activity was greatly affected by land use type. Endo- $\beta-1,3$-glucanase activity were ranged from 136 to 231 Units. $\mathrm{mL}^{-1}$ and protease activity was from 3 to 14 Units. $\mathrm{mL}^{-1}$ in all land types. So there was no significant variation in the activities of endo- $\beta-1,3$-glucanase (Figure $4 \mathrm{~b}$ ) and protease (Figure $4 \mathrm{c}$ ) in different land areas. The 
significant variation of exo- $\beta$-1,4-glucanase activity from highland to lowland suggest the sensitivity of the enzyme as an ecological and a soil quality indicator, so it can be used as a prominent indicator of environmental changes.

Knowledge in relation with soil enzyme activities provides necessary information relating to soil fertility. Land disturbances can negatively affect soil fertility, may leads to the manual addition of soil fertilizers and weedicides, adversely affect soil microbial population. In other words soil functional diversity in some extent is controlled by microbial enzyme activities (Gonnety et al., 2012). Among the three enzyme activities explored, exo- $\beta$-1,4glucanase expressed great variation in their activities in three different geographical land areas. It displayed highest levels of activities in high land areas and the lowest activity was observed in lowland areas. Midland areas showed an activity in between highland and lowland. When considering Kerala's geographical land masses, the anthropogenic activities are increasing from highland to lowland. In this study, exo- $\beta$ - 1,4 -glucanase activity decreased with increasing anthropogenic activities. This trend was not observed in endo- $\beta$ - 1,3 -glucanase and protease. In the light of these observations we can consider exo- $\beta$-1,4-glucanase as a best sensitive indicator for environmental changes. According to Gao et al., 2010, depending on the type of land use, land management and type of enzyme, the absolute enzyme activities may vary in different land areas. Activity of soil enzymes is more related to physical and chemical properties, geology and land uses of soil. Improper land management is obvious in majority of agricultural systems, by the rotating cultivation of crops. Soil quality degradations due to frequent land use changes decline soil microbial biomass and enzyme activities in arable soils compared with natural vegetation (Mganga et al., 2015). Kerala's natural geographical land classification is a best example for changes in environment and land use types. Highland areas are mostly covered with natural vegetation. A combination of natural and agricultural land areas are seen in midlands. But almost all part of lowland areas are arable lands. In this study, the results evidently validates that the sharp declining of number of Streptomyces strains and activity of exo- $\beta$-1,4glucanase enzyme by changing the environment from natural vegetation to arable lands.

\section{Soil pH and Electrical Conductivity (EC):-}

$\mathrm{p}^{\mathrm{H}}$ and EC are the most significant parameters for measuring soil quality. Soil samples collected from three land areas were showed slight variations in $\mathrm{pH}$. Soils under natural vegetation was characterized with high $\mathrm{pH}$ that means they are low acidic soils when compared with agricultural vegetation. $\mathrm{pH}$ values significantly decreased from higher to lower land regions. $\mathrm{pH}$ in highland areas ranged from 7.2 to 6 and in midland areas it was in 6.1 to 5.0 . The highest acidity was noted in lowland regions, where the soils collected from paddy fields ranged from 3.7 to 4.8 . This may be due to the application of herbicides and heavy fertilization of soils over the years for agricultural practices. A significant positive correlation $\left(\mathrm{r}^{2}=0.724\right)$ was shown between soil $\mathrm{pH}$ values and number of Streptomyces strains (Figure 5a). Along with number of Streptomyces isolates $\mathrm{pH}$ values also showed a decreasing trend from highland to low land. Significantly very low $\mathrm{pH}$ was detected in lowland areas. Decrease in $\mathrm{pH}$ was another strong reason for lowering microbial content and soil enzymatic activity. An increase in the concentration of hydrogen ions in soil has a negative effect on its enzymatic activity (Acosta-Martinez and Tabatabai, 2000).

Highest EC was recorded in lowland soils, ranged in between 679 to $885 \mu \mathrm{s} \mathrm{cm}^{-1}$. In midland and highland it was detected at a range of 447 to $635 \mu \mathrm{sm}^{-1}$ and 107 to $352 \mu \mathrm{s} \mathrm{cm}^{-1}$. It gradually decreased from lowland to high land. Number of microbial isolates and activity may be affected by EC. A highly significant negative correlation $\left(\mathrm{r}^{2}=\right.$ 0.908) was shown in between number of Streptomyces isolates and soil EC values (Figure 5b). EC values indicate large accumulation of soluble salts in soils. Negative significant relationship between EC and number of isolates validates that increasing salinity and sodicity tremendously affect the soil Streptomyces community. With increasing salinity microorganisms tend to dehydrate, diminishes microbial growth and activity. Microbial biomass and enzyme activity decreased exponentially with increase in EC (Rietz and Haynes, 2003).

\section{Soil organic carbon and soil organic matter:-}

SOC and SOM percentage was increased with decreasing altitudes. Highest SOC and SOM percentage was reported in lowland areas, unfortunately the area with very less estimated number of Streptomyces isolates (Figure 6). SOC and SOM showed an increasing trend from high land to low land areas, whereas a decreasing trend was obtained in case of number of strains. 3.5 fold increase in SOC and SOM percentage were detected in lowland areas and 2 fold increase in lowland areas when compared with highlands. It may be expected that due to the loss of microbial load in agricultural lands by improper soil management practices reduces the rate of carbon mineralization (Chander et al.,1998). 


\section{Conclusion:-}

The study was conducted to observe how Streptomyces isolates and their enzyme activities fluctuate between the natural geographical land areas in Kerala, highland, midland and lowland. According to this study, large number of Streptomyces strains were isolated from highland areas, the inhabitant of natural forest, compared to other land areas. $\mathrm{pH}$ and EC were highly correlated with the obtaining results, that point out to soil quality. Cumulative anthropogenic activities such as change in land uses especially from forest to rigorous agriculture for sustaining livelihood cause severe consequence on soil quality. Among the three enzymes we have selected for this study, the enzyme exo- $\beta$ - 1,4- glucanase from paddy field isolates showed declining activity than high land and midland isolates. So we can use this enzyme as a most sensitive indicator of environmental changes. Soil Streptomyces biomass and enzyme activities are very sensitive to change in environment and are crucial for maintaining soil quality. In conclusion, unsuitable land use and depletion of nutrients due to the extensive practice of agricultural chemicals influenced soil enzyme activities and microbial dynamics and demonstrated poor soil quality.

\section{Acknowledgement:-}

Authors are grateful to Women Scientist Division, Kerala State Council for Science, Technology and Environment (KSCSTE), Kerala for the financial support.

\section{Reference:-}

1. Acosta-Martinez V. and Tabatabai M. A. (2000): Enzyme activities in a limed agricultural soil. Biol. Fertil. Soils., 31: 85-91.

2. Bielińska, E.J. and Pranagal, J.(2007): Enzymatic Activity of Soil Contaminated with Triazine Herbicides.Polish J. of Environ. Stud., 16(2): 295-300.

3. Bontemps, C., Toussaint, M., Revol, P.V., Hotel, L., Jeanbille, M., Uroz, S., Turpault, M.P., Blaudez, D., Leblond P.(2013): Taxonomic and functional diversity of Streptomyces in a forest soil. FEMS Microbiol. Lett., 342(2): 157-167.

4. Chander, K. Goyal, S., Nandal, D.P.and Kapoor, K. (1998): Soil organic matter, microbial biomass and enzyme activities in a tropical agroforestry system. Biol. Fertil. Soils, 27: 168-172.

5. Gao, Y., Mao, L., Miao, C.Y., Zhou, P., Cao, J.J., Zhi, Y.E. and Shi, W.J. (2010): Spatial characteristics of soil enzyme activities and microbial community structure under different land uses in Choming Island, China: geostatistical modeling and PCR-RAPD method. Sci. Total Environ., 408: 3251-3260.

6. Gonnety, J.T., Assémien, E.F.L., Guéi, A.M., N’Dri, A.A., Djina, Y., Koné A.W. and TondohJ.E. (2012): Effect of land-use types on soil enzymatic activities and chemical properties in semi-deciduous forest areas of Central-West Cote d'Ivoire. Biotechnol. Agron. Soc. Environ., 16(4): 478-485.

7. Hozzein, W.N., Ali, M.I.A. and Rabie, W. (2008): A new preferential medium for enumeration and isolation of desert actinomycetes. World J. Microbiol. Biotechnol.,24(8): 1547-1552.

8. Matson, P.A., Parton, W.J., Power, A.G. and Swift,M.J. (1997): Agricultural Intensification and Ecosystem Properties. Science, 277: 504-509.

9. Mganga, K.Z., Razavi, B.S. and Kuzyakov, Y.(2015): Microbial and enzymes response to nutrient additions in soils of Mt. Kilimanjaro region depending on land use. Eur. J. Soil Biol., 69: 33-40.

10. Miller, G.L. (1959): Use of dinitrosalicylic acid reagent for determination of reducing sugar. Anal. Chem., 31: 426-428.

11. Oliveira, L.R.C., Barbosa, J.B., Martins, M.L.L. and Martins,M.A. (2014): Extracellular production of avicelase by the thermophilic soil bacterium Bacillus sp. SMIA-2. Acta Scienti. Biol. Sci., 36(2): 215-222.

12. Pribyl, D.W.(2010): A critical review of the conventional SOC to SOM conversion factor. Geoderma, 156(3-4): 75-83.

13. Rietz, D.N. and Haynes, R.J.(2003): Effects of irrigation-induced salinity and sodicity on soil microbial activity. Soil Biol. Biochem., 35(6): 845-854.

14. Rupali, D. (2015): Screening and Isolation of protease producing bacteria from soil collected from different areas of Burhanpur Region (MP) India. Int. J Curr. Microbiol. App. Sci., 4(8): 597-606.

15. Schloter, M., Dilly, O. and Munch, J.C. (2003): Indicators for evaluating soil quality. Agric. Ecosyst. Environ., 98(1-3): 255-262.

16. Sharma, M.(2014): Original Research Article Actinomycetes: Source, Identification, and Their Applications. Int. J. Curr. Microbiol. Appl. Sci., 3(2): 801-832.

17. Sinegani, A.A.S. and Mahohi, A. (2010): Soil water potential effects on the cellulase activities of soil treated with sewage sludge. Plant Soil Environ., 56(7): 333-339. 
18. Tate, R.L. (1995): Soil microbiology. Wiley, New York, pp.182-189.

19. Thakur, D., Yadav,A., Gogoi, B.K. and Bora,T.C. (2007): Isolation and screening of Streptomyces in soil of protected forest areas from the states of Assam and Tripura, India, for antimicrobial metabolites.J. Med. Mycol., 17(4): 242-249.

20. Valli, S., Suvathi, S.S., Aysha, O.S., Nirmala, P., Vinoth, K.P. and Reena, A.(2012): Antimicrobial potential of Actinomycetes species isolated from marine environment. Asian Pac. J. Trop. Biomed., 2(6): 469-473.

21. Vranova, V., Rejsek, K. and Formanek, P. (2013): Proteolytic activity in soil: A review. Appl. Soil Ecol., 70: 23-32.

22. Yang, L., Zhang, Y. and Li,F. (2012): Soil enzyme activities and soil fertility dynamics. Advances in Citrus Nutrition, A. K. Srivastava (ed.), Springer Science, pp.143-156.

23. Yeomans, J.C. and Bremner, J.M.(1989): A rapid and precise method for routine determination of organic carbon in soil. Commun. Soil Sci. Plant Anal. 19: 1467-1475.

24. Zhang, X. and Zhang, Y.P. (2013). Cellulases: characteristics, Sources, Production and Applications.Bioprocessing technologies in Biorefinery for Sustainable Production of Fuels, Chemicals, and Polymers, First Edition. Edited by Shang-Tian Yang, Hesham A. El-Enshasy, and Nuttha Thongchul. Published by John Wiley \& Sons, Inc. pp.131-146.

25. Zhou, Y., Wang, X., Wei, W., Xu, J., Wang, W., Xie, Z., Zhang, Z., Jiang, H., Wang, Q. and Wei, C.(2016): A novel efficient $\beta$-glucanase from a paddy soil microbial metagenome with versatile activities. Biotechnol. biofuels, 9:36. 